\title{
Zero-density estimate of $L$-functions attached to Maass forms
}

by

\section{A. Sankaranarayanan and J. Sengupta (Mumbai)}

1. Introduction. Zero-density theorems for $L$-functions to the right of the critical line play a significant role in analytic number theory. These results have been established in various research papers by many mathematicians for different $L$-functions. As a sample we quote a few of them below. Let $L(s)$ be any normalised $L$-function with the first coefficient being 1 , which is absolutely convergent in $\Re s>1$ and satisfies a functional equation of the Riemann zeta-type. We define, for $\sigma \geq 1 / 2$,

$$
N_{L}(\sigma, T):=\#\{\varrho=\beta+i \gamma: L(\varrho)=0, \beta \geq \sigma,|\gamma| \leq T\} .
$$

For the Riemann zeta-function $\zeta(s)$, we know for example the familiar result of Ingham (for $\sigma \geq 1 / 2$ ) that

$$
N_{\zeta}(\sigma, T) \ll T^{3(1-\sigma) /(2-\sigma)}(\log T)^{5} .
$$

In the case of Dirichlet $L$-functions, there is an averaging result of Bombieri (see [1]) which states that when $T \leq Q$,

$$
\sum_{q \leq Q} \sum_{\chi}^{*} N_{\chi}(\sigma, T) \ll T Q^{8(1-\sigma) /(3-2 \sigma)}(\log Q)^{10} .
$$

Here the superscript $*$ means that the sum is over primitive characters. It is also known (see [5] or [21]) that

$$
N_{\zeta}(\sigma, T) \ll T^{12(1-\sigma) / 5}(\log T)^{100} .
$$

We also refer to [3] for sharp density results for the zeros of $\zeta(s)$ in certain ranges of $\sigma$. Some zero-density theorems for $L$-functions can be found in [5], [13], [14] and [21]. As a sample, we quote a result due to Montgomery (see [12]) which we state as

2000 Mathematics Subject Classification: Primary 11F66; Secondary 11M41.

Key words and phrases: L-functions attached to Maass forms, zero-density theorems, mean-square bounds. 
Theorem A. For $T \geq 2$, let

$$
M(T)=\max _{\substack{2 \leq t \leq T \\ \alpha \geq 1 / 2}}|\zeta(\alpha+i t)| .
$$

Then for $3 / 4 \leq \sigma \leq 1$, we have

$$
N_{\zeta}(\sigma, T) \ll\left\{M(5 T)(\log T)^{6}\right\}^{\frac{8(1-\sigma)(3 \sigma-2)}{(4 \sigma-3)(2 \sigma-1)}}(\log T)^{11} .
$$

The central idea is to study how frequently certain Dirichlet polynomials can be large. This main idea was developed and used first by Montgomery (see [12]) and later by many mathematicians (see [3], [8], and [16]). It should be mentioned that zero-density theorems have been established in various situations: for the Dedekind zeta-functions of a number field (see [2]), for the $L$-function attached to a holomorphic cusp form for the full modular group (see [6]), and for the symmetric square $L$-function attached to a holomorphic cusp form for the full modular group (see [20]).

Let $s=\sigma+i t$ denote a complex variable. The parameter $T>0$ will be chosen to be sufficiently large. The letters $C, C^{\prime}$ etc. denote positive constants which are not necessarily the same at each occurrence. Let $f$ denote a normalised (i.e. the first Fourier coefficient is 1) Maass cusp form for $\operatorname{SL}(2, \mathbb{Z})$ which is an eigenfunction of all the Hecke operators $T(n)$ as well as the reflection operator $T_{-1}: z \mapsto-\bar{z}$. We have $T(n) f=\lambda(n) f$ for $n \in \mathbb{N}$, and $\lambda(1)=1$. For $\sigma>1$, we define the standard $L$-function of $f$ as

$$
L(s, f):=\sum_{n=1}^{\infty} \frac{\lambda(n)}{n^{s}}=\prod_{p}\left(1-\lambda(p) p^{-s}+p^{-2 s}\right)^{-1} .
$$

We note that $L(s, f)$ extends as an entire function to the whole complex plane and it satisfies a Riemann zeta-type functional equation under $s \mapsto$ $1-s$ (see [7]). We also note that $N_{L}(\sigma, T) \ll T \log T$ for $1 / 2 \leq \sigma \leq 1 / 2+$ $1 / \log T$. The zero-density estimates to the right of the critical line in the case of the standard $L$-function attached to a normalised Maass cusp form are of great interest and seem to be unavailable in the literature.

The main aim of this paper is to prove

Theorem 1. For $\sigma \geq 1 / 2+1 / \log T$, we have

$$
N_{L}(\sigma, T) \ll T^{4(1-\sigma) /(3-2 \sigma)}(\log T)^{26},
$$

where the implied constant depends on $f$.

As an application, we can extend Theorem 1 of [19] by Ramachandra and the first author. Precisely, we can prove the local theorem on the zeros of $L(s, f)$ in the neighbourhood of the critical line: 
THEOREm 2. If $L(s, f) \neq 0$ in the rectangle

$$
\left\{\frac{1}{2}+\frac{1}{10 \log \log T}<\sigma \leq 1, T-H \leq t \leq T+H\right\}
$$

with $H=C \log \log \log T, T \geq 100$, then there is at least one zero of $L(s, f)$ in the disc of radius $C^{\prime}(\log \log T)^{-1}$ with centre $1 / 2+i T$. Here $C, C^{\prime}$ are effective positive constants depending on $f$.

REMARK. The zero-counting argument adapted in this paper is somewhat familiar (see for example [17] and [20]). However, the real difficulty in this situation lies in getting certain mean-value estimates of the zero-detector function $F_{2}(s)$ on certain lines in terms of precise log powers. For this, we need to first establish upper bounds on the discrete mean involving certain arithmetical functions. We prove these estimates in a sequence of lemmas in Section 3.

Acknowledgements. The authors are grateful to Professor Jeffrey Hoffstein for useful e-mail discussions. The authors are also indebted to the anonymous referee for some helpful comments.

2. Notation and preliminaries. The letters $C, A$ and $B$ (with or without subscripts) denote effective positive constants unless otherwise specified. They need not be the same at every occurrence. Throughout the paper we assume $T \geq T_{0}$ where $T_{0}$ is a large positive constant. We write $f(x) \ll g(x)$ to mean $|f(x)|<C_{1} g(x)$ for $x \geq x_{0}$ where $C_{1}$ is some absolute positive constant (sometimes we denote this by the $O$ notation also). Let $s=\sigma+i t$ and $w=u+i v$. The implied constants are all effective but they will depend on the form $f$ in question.

For $\sigma>1$, let

$$
\frac{1}{L(s, f)}=\sum_{n=1}^{\infty} \frac{\mu^{*}(n)}{n^{s}} .
$$

Then $\mu^{*}(n)$ is a multiplicative function and its values on prime powers are as follows:

$$
\mu^{*}\left(p^{a}\right)= \begin{cases}1 & \text { if } a=0 \\ -\lambda(p) & \text { if } a=1 \\ 1 & \text { if } a=2 \\ 0 & \text { if } a \geq 3\end{cases}
$$

We keep in mind that $\mu^{*}(n)=0$ unless $n$ is cube-free. 


\section{Some lemmas}

LEMMA 3.1. We have the estimate

$$
\sum_{n \leq x}|\lambda(n)|^{4} \ll x \log x .
$$

Proof. Let $L_{4}(s)=\sum_{n=1}^{\infty}(\lambda(n))^{4} / n^{s}$. There is a dominant Dirichlet series $L_{4}^{*}(s)$ with positive coefficients $\lambda^{*}(n)$ which has the property that

$$
\sum_{n \leq x}|\lambda(n)|^{4} \leq \sum_{n \leq x} \lambda^{*}(n)
$$

(see for example [4]). In fact, $L_{4}^{*}(s)$ has a pole of order 2 at $s=1$, is otherwise analytic and is given by

$$
L_{4}^{*}(s)=L\left(s, f, \vee^{4}\right)\left(L\left(s, f, \vee^{2}\right)\right)^{3}(\zeta(s))^{2} .
$$

The two functions $L\left(s, f, \vee^{4}\right)$ and $L\left(s, f, \vee^{2}\right)$ on the right hand side are respectively the symmetric fourth and symmetric square $L$-series associated to $f$. As the analytic continuation and functional equations of these series are known (see for example [9], [10]), it follows from a standard Tauberian argument that

$$
\sum_{n \leq x} \lambda^{*}(n) \asymp C x \log x
$$

where the constant $C$ depends on $f$. Now, the lemma follows from (3.1).

LEMMA 3.2. We have the estimate

$$
\sum_{l \leq x} \frac{\left(\mu^{*}(l)\right)^{2}}{l} \ll \log x .
$$

Proof. We note that $\mu^{*}(l)=0$ unless $l$ is cube-free. So we write $l=d_{1}^{2} d_{2}$ with $\left(d_{1}, d_{2}\right)=1$ and $d_{1}, d_{2}$ square-free. Then

$$
\left(\mu^{*}(l)\right)^{2}=\left(\mu^{*}\left(d_{1}^{2}\right)\right)^{2}\left(\mu^{*}\left(d_{2}\right)\right)^{2}=\left(\lambda\left(d_{2}\right)\right)^{2} .
$$

Using (3.2), we have

$$
\begin{aligned}
\sum_{l \leq x} \frac{\left(\mu^{*}(l)\right)^{2}}{l} & =\sum_{\substack{d_{1}^{2} d_{2} \leq x \\
\left(d_{1}, d_{2}\right)=1 \\
d_{1}, d_{2} \text { square-free }}} \frac{\left(\lambda\left(d_{2}\right)\right)^{2}}{d_{1}^{2} d_{2}} \\
& =\sum_{d_{1}^{2} \leq x} \frac{1}{d_{1}^{2}} \sum_{d_{2} \leq x d_{1}^{-2}} \frac{\left(\lambda\left(d_{2}\right)\right)^{2}}{d_{2}} \ll(\log x)\left(\sum_{d_{1}^{2} \leq x} \frac{1}{d_{1}^{2}}\right) \\
& \ll \log x,
\end{aligned}
$$

since $\sum_{m \leq Y}(\lambda(m))^{2} \ll Y($ see $[7])$. 
LEMMA 3.3. We have the estimate

$$
\sum_{l \leq x} \frac{\left(\mu^{*}(l)\right)^{4}}{l} \ll(\log x)^{2} .
$$

Proof. From (3.2), we observe that

$$
\begin{aligned}
\sum_{l \leq x} \frac{\left(\mu^{*}(l)\right)^{4}}{l} & =\sum_{\substack{d_{1}^{2} d_{2} \leq x \\
\left(d_{1}, d_{2}\right)=1 \\
d_{1}, d_{2} \text { square-free }}} \frac{\left(\mu^{*}\left(d_{1}^{2} d_{2}\right)\right)^{4}}{d_{1}^{2} d_{2}} \\
& \leq \sum_{d_{1}^{2} d_{2} \leq x} \frac{\left(\lambda\left(d_{2}\right)\right)^{4}}{d_{1}^{2} d_{2}}=\sum_{d_{1}^{2} \leq x} \frac{1}{d_{1}^{2}} \sum_{d_{2} \leq x d_{1}^{-2}} \frac{\left(\lambda\left(d_{2}\right)\right)^{4}}{d_{2}} \\
& \ll(\log x)^{2},
\end{aligned}
$$

on using the estimate in Lemma 3.1.

LEMMA 3.4. Let

$$
c(n)=\sum_{\substack{d \mid n \\ d \leq T}} \mu^{*}(d) \lambda(n / d)
$$

Then

$$
\sum_{n \leq x}|c(n)|^{2} \ll x(\log x)^{17} .
$$

Proof. For any $a_{i} \in \mathbb{R}$ and $m \in \mathbb{N}$, we have

$$
\left(\sum_{i=1}^{m} a_{i}\right)^{2} \leq m^{2} \sum_{i=1}^{m} a_{i}^{2}
$$

Therefore (with $\tau(n)$ being the number of positive divisors of $n$ ),

$$
(c(n))^{2} \leq(\tau(n))^{2} \sum_{\substack{d \mid n \\ d \leq T}}\left(\mu^{*}(d)\right)^{2}(\lambda(n / d))^{2} .
$$

Hence we have

$$
\begin{aligned}
S & :=\sum_{n \leq x}(c(n))^{2} \leq \sum_{l m \leq x}(\tau(l m))^{2}\left(\mu^{*}(l)\right)^{2}(\lambda(m))^{2} \\
& \leq \sum_{l \leq x} \sum_{m \leq x l^{-1}}(\tau(l))^{2}(\tau(m))^{2}\left(\mu^{*}(l)\right)^{2}(\lambda(m))^{2} \\
& =\sum_{l \leq x}(\tau(l))^{2}\left(\mu^{*}(l)\right)^{2} \sum_{m \leq x l^{-1}}(\tau(m))^{2}(\lambda(m))^{2} \\
& \leq \sum_{l \leq x}(\tau(l))^{2}\left(\mu^{*}(l)\right)^{2}\left\{\left(\sum_{m \leq x l^{-1}}(\tau(m))^{4}\right)^{1 / 2}\left(\sum_{m \leq x l^{-1}}(\lambda(m))^{4}\right)^{1 / 2}\right\} .
\end{aligned}
$$


Since $(\tau(m))^{4} \leq \tau_{2^{4}}(m)$ (where $\tau_{j}(n)$ denotes the $j$-fold divisor function), we have

$$
\sum_{m \leq x l^{-1}}(\tau(m))^{4} \ll \frac{x}{l}(\log x)^{15} .
$$

Now, using Lemma 3.1, we note that the term within the curly bracket above is $\ll(x / l)(\log x)^{8}$. Thus, we obtain

$$
\begin{aligned}
S & \ll x(\log x)^{8} \sum_{l \leq x} \frac{(\tau(l))^{2}\left(\mu^{*}(l)\right)^{2}}{l} \\
& \ll x(\log x)^{8}\left(\sum_{l \leq x} \frac{(\tau(l))^{4}}{l}\right)^{1 / 2}\left(\sum_{l \leq x} \frac{\left(\mu^{*}(l)\right)^{4}}{l}\right)^{1 / 2} .
\end{aligned}
$$

Now, using Lemma 3.3, we get

$$
\sum_{n \leq x}(c(n))^{2} \ll x(\log x)^{17}
$$

Lemma 3.5 (Montgomery-Vaughan). If $h_{n}$ is an infinite sequence of complex numbers such that $\sum_{n=1}^{\infty} n\left|h_{n}\right|^{2}$ is convergent, then

$$
\int_{T}^{T+H}\left|\sum_{n=1}^{\infty} h_{n} n^{-i t}\right|^{2} d t=\sum_{n=1}^{\infty}\left|h_{n}\right|^{2}(H+O(n)) .
$$

Proof. See for example Lemma 3.3 of [15], or [18].

Lemma 3.6. If $N_{L}(\sigma, T, T+1)$ denotes the number of zeros $\varrho=\beta+i \gamma$ of $L(s, f)$ with $\beta \geq \sigma, T \leq \gamma<T+1$, then

$$
N_{L}(\sigma, T, T+1) \ll \log T .
$$

Proof. We define

$$
F_{1}(s)=\frac{L(s, f)}{\prod_{\varrho}\left(1-\frac{s-s_{0}}{\varrho-s_{0}}\right)}
$$

where $\varrho$ in the product runs over the zeros $\varrho=\beta+i \gamma$ of $L(s, f)$ with $0 \leq \beta \leq 1$ and $T<\gamma<T+1$ and $s_{0}=\sigma_{0}+i \gamma$ with $\sigma_{0}$ sufficiently large. We note that

$$
\begin{aligned}
\left|F_{1}\left(s_{0}\right)\right| & =\left|L\left(s_{0}, f\right)\right| \geq 1-\sum_{n=2}^{\infty} \frac{|\lambda(n)|}{n^{\sigma_{0}}} \\
& \geq 2-\left(\sum_{n=1}^{\infty} \frac{|\lambda(n)|^{2}}{n^{\sigma_{0}}}\right)^{1 / 2}\left(\zeta\left(\sigma_{0}\right)\right)^{1 / 2} \geq C
\end{aligned}
$$

for sufficiently large $\sigma_{0}$ which may depend upon $f$ (note that both the series $\sum_{n=1}^{\infty}|\lambda(n)|^{2} n^{-\sigma_{0}}$ and $\zeta\left(\sigma_{0}\right)$ approach 1 as $\left.\sigma_{0} \rightarrow \infty\right)$. Here $C$ is a certain 
positive constant. For $\left|s-s_{0}\right|=3 \sigma_{0}$, we have

$$
\left|1-\frac{s-s_{0}}{\varrho-s_{0}}\right| \geq\left|\frac{s-s_{0}}{\varrho-s_{0}}\right|-1 \geq \frac{3 \sigma_{0}}{\sigma_{0}-\beta}-1 \geq 2 .
$$

This implies that

$$
C<\left|F_{1}\left(s_{0}\right)\right|<\max _{\left|s-s_{0}\right|=3 \sigma_{0}}\left|F_{1}(s)\right|<\max _{\left|s-s_{0}\right| \leq 3 \sigma_{0}} \frac{|L(s, f)|}{2^{N}} \ll \frac{T^{C}}{2^{N}}
$$

and hence we obtain the lemma.

Lемма 3.7. For $\sigma>1$, define

$$
F_{2}(s):=L(s, f) \sum_{n \leq T} \frac{\mu^{*}(n)}{n^{s}}-1=L(s, f) M_{T}(s)-1=: \sum_{n=1}^{\infty} \frac{c(n)}{n^{s}} .
$$

Then

$$
c(n)=\sum_{\substack{d \mid n \\ d \leq T}} \mu^{*}(d) \lambda(n / d)
$$

and for $\sigma>1$,

$$
F_{2}(s)=\sum_{n>T} c(n) / n^{s}
$$

Proof. First we observe that

$$
\sum_{d \mid n} \mu^{*}(d) \lambda(n / d)= \begin{cases}1 & \text { if } n=1 \\ 0 & \text { if } n \geq 2\end{cases}
$$

Now, we define

$$
a(d):= \begin{cases}\mu^{*}(d) & \text { if } d \leq T \\ 0 & \text { if } d>T\end{cases}
$$

From the definition of $F_{2}(s)$, we notice that

$$
c(n)=\sum_{d \mid n} a(d) \lambda(n / d)-\sum_{d \mid n} \mu^{*}(d) \lambda(n / d) .
$$

If $n \leq T$, then $d \leq T$ (since $d$ is a divisor of $n$ ) so that $a(d)=\mu^{*}(d)$. Therefore $c(n)=0$ for $n \leq T$. For $n>T$, the second sum in (3.5) is zero and hence from (3.4), we get (3.3).

\section{Proof of the theorems}

Proof of Theorem 1. By using dyadic partitions, it is enough to prove the theorem for $T \leq \gamma \leq 2 T$. We divide the rectangle bounded by the lines with real parts $\sigma, 1$ and imaginary parts $T, 2 T$ into abutting smaller rectangles of height $2(\log T)^{2}$. From Lemma 3.6, the multiplicity of any zero $\varrho$ of $L(s, f)$ is $\ll \log T$. Therefore, without loss, we can assume that the zeros are simple 
in the counting process. We count the number of those smaller rectangles of height $2(\log T)^{2}$ which contain at least one zero and multiply by $C(\log T)^{3}$ to get a bound for $N_{L}(\sigma, T, 2 T)$.

We define the zero-detector function

$$
F_{2}(s):=L(s, f) \sum_{n \leq T} \frac{\mu^{*}(n)}{n^{s}}-1=L(s, f) M_{T}(s)-1=: \sum_{n>T} \frac{c(n)}{n^{s}} .
$$

From Lemma 3.7, we notice that

$$
c(n)=\sum_{\substack{d \mid n \\ d \leq T}} \mu^{*}(d) \lambda(n / d)
$$

For any fixed zero $\varrho=\beta+i \gamma$, we let

$$
G(s)=F_{2}(s) Y^{s-\varrho} e^{(s-\varrho)^{2}}
$$

where $Y$ is a parameter satisfying $T^{-A} \leq Y \leq T^{A}$. We select one zero $\varrho_{j}$ in each of the rectangles (for $j=1,2, \ldots$ )

$$
\left\{\frac{1}{2}+\frac{1}{\log T} \leq \sigma \leq 1, T+2(j-1)(\log T)^{2} \leq t \leq T+2 j(\log T)^{2}\right\} .
$$

We partition these rectangles into odd and even ones. Note that for any two zeros $\varrho, \varrho^{\prime}$ in two even (respectively odd) rectangles, we have $\left|\gamma-\gamma^{\prime}\right| \geq$ $2(\log T)^{2}$. Let $\mathcal{A}$ and $\mathcal{B}$ denote the sets of the chosen zeros corresponding to the sets of odd and even rectangles respectively. Let $\varrho \in \mathcal{A}$ be any typical chosen zero. By Cauchy's residue theorem, we have

$$
\left|\frac{1}{2 \pi i} \int_{R(\varrho)} \frac{G(s)}{s-\varrho} d s\right|=1
$$

where the integral is taken over the rectangle $R(\varrho)$ defined by

$$
R(\varrho):=\left\{\frac{1}{2} \leq \sigma \leq 1+\frac{1}{\log T},|t-\gamma| \leq B(\log T)^{2}\right\} .
$$

Here $1 / 4 \leq B \leq 1$ is chosen such that the horizontal sides of $R(\varrho)$ are free from zeros of $L(s, f)$. If $Y$ is chosen to satisfy $T^{-A} \leq Y \ll T^{A}$, then the contributions from the horizontal sides of $R(\varrho)$ to the integral are $O\left(T^{-10}\right)$ owing to the exponentially decaying factor $e^{(s-\varrho)^{2}}$. We denote the vertical sides of $R(\varrho)$ by $V_{1}$ and $V_{2}$ so that we have

$$
\begin{aligned}
1= & O\left(\log T\left(\int_{V_{1}}\left|F_{2}(s)\right| d t\right) Y^{1 / 2-\beta}\right. \\
& \left.+\log T\left(\int_{V_{2}}\left|F_{2}(s)\right| d t\right) Y^{1+1 / \log T-\beta}\right)
\end{aligned}
$$




$$
\begin{aligned}
= & O\left(\log T\left(1+\int_{V_{1}}\left|F_{2}(s)\right| d t\right) Y^{1 / 2-\beta}\right. \\
& \left.+\log T\left(T^{-10}+\int_{V_{2}}\left|F_{2}(s)\right| d t\right) Y^{1+1 / \log T-\beta}\right) .
\end{aligned}
$$

We choose $Y$ such that

$$
Y^{1 / 2-\beta}\left(1+\int_{V_{1}}\left|F_{2}(s)\right| d t\right)=Y^{1-\beta}\left(T^{-10}+\int_{V_{2}}\left|F_{2}(s)\right| d t\right) .
$$

Let

$$
\begin{aligned}
& J_{1}(\varrho)=1+\int_{V_{1}}\left|F_{2}(s)\right| d t \\
& J_{2}(\varrho)=T^{-10}+\int_{V_{2}}\left|F_{2}(s)\right| d t .
\end{aligned}
$$

We notice that (from Lemmas 3.2 and 3.5)

$$
\int_{T}^{2 T}\left|M_{T}(1 / 2+i t)\right|^{2} d t=\sum_{n \leq T} \frac{\left|\mu^{*}(n)\right|^{2}}{n}(T+O(n)) \ll T \log T .
$$

From (5.7) of [11], we have

$$
\int_{T}^{2 T}|L(1 / 2+i t)|^{2} d t \ll T \log T .
$$

Therefore by the Cauchy-Schwarz inequality, we find that

$$
\int_{T}^{2 T}\left|F_{2}(1 / 2+i t)\right| d t \ll T \log T
$$

and using Lemma 3.5 (Montgomery-Vaughan theorem) and the estimate in Lemma 3.4, we have

$$
\begin{aligned}
\int_{T}^{2 T}\left|F_{2}(1+1 / \log T+i t)\right|^{2} d t & =\sum_{n>T} \frac{|c(n)|^{2}}{n^{2+2 / \log T}}(T+O(n)) \\
& \ll \sum_{n>T} \frac{|c(n)|^{2}}{n^{1+2 / \log T}}=\int_{T}^{\infty} \frac{d\left(\sum_{n \leq u}|c(n)|^{2}\right)}{u^{1+2 / \log T}} \\
& \ll(\log T)^{19},
\end{aligned}
$$

on integrating by parts.

Note that (from (4.2) and (4.3))

$$
Y=\left(\frac{J_{1}}{J_{2}}\right)^{2} \geq \frac{1}{T^{-10}+T^{C}}, \quad Y \leq \frac{T^{C}}{T^{-10}},
$$


so that the condition on $Y$ is satisfied. Hence we have

$$
1 \leq 2 C(\log T)\left(\frac{J_{1}}{J_{2}}\right)^{2(1-\beta)} J_{2}=2 C(\log T) J_{1}^{2(1-\beta)} J_{2}^{2 \beta-1} .
$$

It follows from the above that

$$
\sum_{\varrho \in \mathcal{A}} J_{1}(\varrho) \ll T \log T \text { and } \sum_{\varrho \in \mathcal{A}}\left(J_{2}(\varrho)\right)^{2} \ll(\log T)^{21} .
$$

The same argument is applicable to the zeros in the set $\mathcal{B}$. Thus we obtain

$$
\sum_{\varrho \in \mathcal{A}} J_{1}(\varrho)+\sum_{\varrho \in \mathcal{B}} J_{1}(\varrho)=\sum_{\varrho \in \mathcal{A} \cup \mathcal{B}} J_{1}(\varrho) \ll T \log T
$$

and similarly

$$
\sum_{\varrho \in \mathcal{A}}\left(J_{2}(\varrho)\right)^{2}+\sum_{\varrho \in \mathcal{B}}\left(J_{2}(\varrho)\right)^{2}=\sum_{\varrho \in \mathcal{A} \cup \mathcal{B}}\left(J_{2}(\varrho)\right)^{2} \ll(\log T)^{21}
$$

and so

$$
\begin{aligned}
& \#\left\{\varrho: J_{1}(\varrho) \geq W_{1}\right\} \leq A \frac{T \log T}{W_{1}}, \\
& \#\left\{\varrho: J_{2}(\varrho) \geq W_{2}\right\} \leq A \frac{(\log T)^{21}}{W_{2}^{2}} .
\end{aligned}
$$

Now we fix $W_{1}=W_{2}^{2} T$. Hence the total number of zeros coming from the two sets in (4.4) is at most

$$
A(\log T)^{21}\left\{\frac{T}{W_{1}}+\frac{1}{W_{2}^{2}}\right\} .
$$

From (4.1), for the remaining zeros, we have

$$
J_{1}(\varrho)<W_{1} \quad \text { and } \quad J_{2}(\varrho)<W_{2}
$$

and also

$$
\begin{aligned}
3 / 4 & \leq 2 C(\log T) W_{1}^{2(1-\beta)} W_{2}^{2 \beta-1} \\
& =2 C(\log T) W_{1}^{2(1-\sigma)} W_{1}^{2(\sigma-\beta)} W_{2}^{2 \sigma-1} W_{2}^{2(\beta-\sigma)} \\
& =2 C(\log T) W_{1}^{2(1-\sigma)} W_{2}^{2 \sigma-1}\left(\frac{W_{2}}{W_{1}}\right)^{2(\beta-\sigma)} \\
& =2 C(\log T) W_{1}^{2(1-\sigma)} W_{2}^{2 \sigma-1}\left(\frac{1}{W_{2} T}\right)^{2(\beta-\sigma)} .
\end{aligned}
$$

Suppose that $W_{2}>1 / T$ and so $\left(1 / W_{2} T\right)^{2(\beta-\sigma)} \leq 1$. Then we get

$$
\begin{aligned}
3 / 4 & \leq 2 C(\log T) W_{1}^{2(1-\sigma)} W_{2}^{2 \sigma-1} \\
& =2 C(\log T)\left(W_{2}^{2} T\right)^{2(1-\sigma)} W_{2}^{2 \sigma-1}=2 C(\log T) T^{2(1-\sigma)} W_{2}^{3-2 \sigma} .
\end{aligned}
$$


We choose

$$
W_{2}=(4 C \log T)^{-\frac{1}{3-2 \sigma}} T^{-\frac{2(1-\sigma)}{3-2 \sigma}} .
$$

Clearly $W_{2}>T^{-1}$. For this choice of $W_{2},(4.5)$ implies that $3 / 4 \leq 1 / 2$, which is absurd; this means that we should count only those zeros which satisfy (4.4). Hence we get

$$
N_{L}(\sigma, T, 2 T) \ll \frac{(\log T)^{21}}{W_{2}^{2}}(\log T)^{3} \ll T^{4(1-\sigma) /(3-2 \sigma)}(\log T)^{26},
$$

which proves the theorem.

Proof of Theorem 2. The proof is entirely similar to the proof of Theorem 1 of [19] and hence is omitted.

\section{References}

[1] E. Bombieri, On the large sieve, Mathematika 12 (1965), 201-225.

[2] D. R. Heath-Brown, On the density of the zeros of the Dedekind zeta-function, Acta Arith. 33 (1977), 169-181.

[3] - Zero density estimates for the Riemann zeta-function and Dirichlet L-functions, J. London Math. Soc. (2) 19 (1979), 221-232.

[4] J. Hoffstein and P. Lockhart, Coefficients of Maass forms and the Siegel zero, Ann. of Math. 140 (1994), 161-181.

[5] A. Ivić, The Riemann Zeta-Function, Wiley, 1985.

[6] -, On zeta-functions associated with Fourier coefficients of cusp forms, in: Proc. Amalfi Conf. on Analytic Number Theory (Maiori, 1989), E. Bombieri et al. (eds.), Univ. di Salerno, 1992, 231-246.

[7] H. Iwaniec, Introduction to the Spectral Theory of Automorphic Forms, Rev. Mat. Iberoamer., Madrid, 1995.

[8] M. Jutila, Zero density estimates for L-functions, Acta Arith. 32 (1977), 55-62.

[9] H. H. Kim and F. Shahidi, Cuspidality of symmetric powers with applications, Duke Math. J. 112 (2002), 177-197.

[10] - - - Functorial products for $\mathrm{GL}_{2} \times \mathrm{GL}_{3}$ and the symmetric cube for $\mathrm{GL}_{2}$, Ann. of Math. (2) 155 (2002), 837-893.

[11] W. Kohnen, A. Sankaranarayanan and J. Sengupta, The quadratic mean of automorphic L-functions, in: Automorphic Forms and Zeta Functions, S. Böcherer et al. (eds.), World Sci., 2005, 262-279.

[12] H. L. Montgomery, Mean and large values of Dirichlet polynomials, Invent. Math. 8 (1969), 334-345.

[13] -, Zeros of L-functions, ibid., 346-354.

[14] - , Topics in Multiplicative Number Theory, Springer, Berlin, 1971.

[15] H. L. Montgomery and R. C. Vaughan, Hilbert's inequality, J. London Math. Soc. (2) 8 (1974), 73-82.

[16] K. Ramachandra, Some new density estimates for the zeros of the Riemann zetafunction, Ann. Acad. Sci. Fenn. Ser AI 1 (1975), 177-182.

[17] —, Riemann Zeta-Function, Ramanujan Inst. Publ., Madras Univ., Chennai, 1979.

[18] —, Some remarks on a theorem of Montgomery and Vaughan, J. Number Theory 11 (1979), 465-471. 
[19] K. Ramachandra and A. Sankaranarayanan, On some theorems of Littlewood and Selberg, IV, Acta Arith. 70 (1995), 79-84.

[20] A. Sankaranarayanan, Fundamental properties of symmetric square L-functions, II, Funct. Approx. Comment. Math. 30 (2002), 89-115.

[21] E. C. Titchmarsh, The Theory of the Riemann Zeta-Function, 2nd ed., edited by D. R. Heath-Brown, Clarendon Press, Oxford, 1986.

School of Mathematics

Tata Institute of Fundamental Research

Homi Bhabha Road

Mumbai 400 005, India

E-mail: sank@math.tifr.res.in sengupta@math.tifr.res.in

Received on 7.6.2006

and in revised form on 18.12.2006 